\title{
Compact forms of reduced density matrices
}

\author{
L. M. Tel, E. Pérez-Romero, F. J. Casquero, and C. Valdemoro* \\ Departamento de Química-Física, Universidad de Salamanca, 37008-Salamanca, Spain
}

(Received 13 January 2003; published 21 May 2003)

\begin{abstract}
The objective of this paper is to identify the necessary and sufficient minimum information carried by Reduced Density Matrices (RDMs). This minimum of essential information determines a set of equivalent compact-form matrices that are devoid of the redundant information present in the original RDMs when the system possesses symmetry invariances. The basic properties and algebra operations of these compact-form matrices are obtained here. The use of these new mathematical objects renders far more economical the operations and storage of RDMs and will thus be of interest in the study of systems with symmetry invariances. In particular, it opens the possibility of using large basis for medium-sized systems when solving iteratively the contracted Schrödinger equation.
\end{abstract}

DOI: 10.1103/PhysRevA.67.052504

PACS number(s): $31.15 . \mathrm{Hz}, 31.10 .+\mathrm{z}$

\section{INTRODUCTION}

The reduced density matrices (RDMs) [1-5] are mathematical-physical objects that carry the information about the electronic structure of an $N$-electron system in a far more economical way than the wave function from which they are derived. However, as the size of the system increases, the size of the basis of representation of these matrices also increases and, therefore, the dimensions of these matrices become too large for easy handling.

On the other hand, it is well known [6] that the RDMs have a much smaller number of independent parameters than the full configuration interaction (FCI) wave function. However, there are several means of rendering the information contained in the RDMs more compact. A simple way of reducing the number of independent elements can be to take advantage of the characteristic properties of the RDMs, such as its Hermiticity. Another possibility is to shrink the dimensions of the RDMs by labeling rows and columns with sets of ordered indices. The information contained in a $p-\mathrm{RDM}$ is still carried out by $\left(\left(_{2}^{2 K}{ }_{2}\right)+1\right.$ ) for a one-electron function space of dimension $2 K$. This number is further reduced because of the requirement of fixed trace and $N$-representability conditions. The use of a symmetry-adapted basis of spin orbitals, geminals, etc., makes many of their elements vanish. This kind of study has been carried out by several authors mainly in the coordinate representation, particularly making use of the spin-symmetry properties of the RDMs [7].

However, in many cases it may be preferable to work with an atomic basis instead of with a symmetry-adapted molecular or crystal-adapted basis, and it may be necessary to keep a simple non-spin-adapted labeling in the RDM rows and columns. This is the case on solving the contracted Schrödinger equation (CSE) [8-14], which involves the simultaneous use of three different orders of RDMs, the need for performing contractions of the 3- and 4-RDMs, and the reconstruction of higher-order RDM elements in terms of the lower-order ones.

\footnotetext{
*Present address: Instituto de Matemáticas y Física Fundamental, CSIC, Serrano 123, 28006 Madrid, Spain.
}

The aim of this paper is to report a method for obtaining the same information as a $p$-RDM although in a compact form from which all the redundant information has been omitted. This methodology takes into account the invariants of the strings of creation-annihilation fermion operators in the second quantization because their expectation values are the RDM elements. Therefore, our purpose is to render feasible the operations with RDMs, using as few matrix elements as possible, without necessarily being forced to use symmetry or spin-adapted basis functions, but taking the utmost advantage of the symmetry of the system.

In the following section, the notation and the necessary background information are given. The procedure to calculate the number of invariant elements of a given $p$-RDM is described in some detail in Sec. III. The construction of the compact-form matrices, equivalent to the RDMs, is shown in Sec. IV. Finally, in Sec. V, the handling rules for operating with these compact-form matrices are reported.

\section{NOTATION AND THEORETICAL BACKGROUND}

\section{A. Transformation of the basis set}

Numerical solutions of the electronic problems in atoms or molecules are usualy achieved by developing wave functions and operators in a finite set of spin orbitals. In the present work, orthonormality of the basis will always be assumed. This basis set of orbitals may be modified by a set of transformations $\hat{R}$, such as rotations or reflections, of the coordinate system. If the set of $2 K$ spin orbitals is stable under a linear operation $\hat{R}$, the new orbitals are expressed as linear combinations of the old ones. In a different basis of representation, the spin-orbital labels will be indicated by primed latin alphabets, and so we will have

$$
\varphi_{p^{\prime}}=\hat{R} \phi_{r}=\sum_{k}^{2 K} \phi_{k} R_{k ; p^{\prime}}, \quad \phi_{r}=\sum_{i^{\prime}}^{2 K} \varphi_{i^{\prime}} R_{i^{\prime} ; r}^{\dagger} .
$$

The transformation matrix of dimensions $2 K \times 2 K$ is unitary and thus preserves the orthonormality of the set. $\mathbf{R}^{\dagger}$ is the matrix associated to the inverse transformation. 
The set of all those transformation matrices forms a group $\mathrm{U}(2 K)$. Some subgroups are $\mathrm{U}(K)$ if no spin mixing is allowed, and $\mathrm{O}(K)$ if orbitals are restricted to be real. Other subgroups are those related to the spatial symmetry of the problem, such as a symmetry transformation or a transformation to a symmetry-adapted basis.

The change of basis modifies according to the creationannihilation operators [15]

$$
\begin{array}{ll}
b_{i^{\prime}}^{\dagger}=\hat{R} b_{i}^{\dagger} \hat{R}^{-1}=\sum_{k}^{2 K} b_{k}^{\dagger} R_{k ; i^{\prime}}, & b_{k}^{\dagger}=\sum_{i^{\prime}}^{2 K} b_{i^{\prime}}^{\dagger} R_{i^{\prime} ; k}^{\dagger}, \\
b_{i^{\prime}}=\hat{R} b_{i} \hat{R}^{-1}=\sum_{k}^{2 K} b_{k} R_{i^{\prime} ; k}^{\dagger}, \quad b_{k}=\sum_{i^{\prime}}^{2 K} b_{i^{\prime}} R_{k ; i^{\prime}},
\end{array}
$$

where invariance of the vacuum has been assumed. Creation operators transform as the spin orbitals themselves, while the matrices for the annihilators are complex conjugates to those for the creators.

The number operator is independent of the choice of the basis set:

$$
\hat{N}=\sum_{i^{\prime}=1}^{2 K} b_{i^{\prime}}^{\dagger} b_{i^{\prime}}=\sum_{k=1}^{2 K} b_{k}^{\dagger} b_{k} .
$$

The transformation of the spin orbitals also transforms the string of two creation and annihilation operators. Thus, the creation operators transform as

$$
\begin{aligned}
b_{i^{\prime}}^{\dagger} b_{j^{\prime}}^{\dagger}=\hat{R} b_{i}^{\dagger} b_{j}^{\dagger} \hat{R}^{-1} & =\hat{R} b_{i}^{\dagger} \hat{R}^{-1} \hat{R} b_{j}^{\dagger} \hat{R}^{-1}=\sum_{k, l}^{2 K} b_{k}^{\dagger} b_{l}^{\dagger} R_{k ; i^{\prime}} R_{l ; j^{\prime}} \\
& =\sum_{k, l}^{2 K} b_{k}^{\dagger} b_{l}^{\dagger 2} R_{k l ; i^{\prime} j^{\prime}} \quad\left(\forall i^{\prime}, j^{\prime}\right) .
\end{aligned}
$$

In order to have uniquely defined two-electron or geminal functions, the basis set must also be uniquely defined; this is achieved by imposing an ordering of the operator labels or, equivalently, an antisymmetrized product of the one-electron basis. It transforms as

$$
b_{i^{\prime}}^{\dagger} b_{j^{\prime}}^{\dagger}=\sum_{k<l}^{2 K} b_{k}^{\dagger} b_{l}^{\dagger}{ }^{2} \mathcal{R}_{k l ; i^{\prime} j^{\prime}} \quad\left(i^{\prime}<j^{\prime}\right),
$$

where ${ }^{2} \mathcal{R}$ is the matrix transformation of the two-particle operators. It is the antisymmetric part of the $\mathbf{R} \otimes \mathbf{R}$ matrix product. For any transformation $\hat{R}$,

$$
{ }^{2} \mathcal{R}_{k l ; i j}=R_{k ; i} R_{l ; j}-R_{l ; i} R_{k ; j}(k<l ; i<j) .
$$

Both matrices, ${ }^{2} \mathbf{R}$ and ${ }^{2} \mathcal{R}$, of dimensions $K^{2} \times K^{2}$ and $\left(\begin{array}{c}2 K \\ 2\end{array}\right) \times\left({ }_{2}^{2 K}\right)$, respectively, are also unitary:

$$
{ }^{2} \mathbf{R}^{-1}={ }^{2} \mathbf{R}^{\dagger},{ }^{2} \mathcal{R}-1={ }^{2} \mathcal{R}^{\dagger} .
$$

Hence, the global transformation of a string of several creation operators is closely related to that of the spin orbitals.
The transformation of a string of two annihilators takes one of these alternative forms

$$
\begin{aligned}
& \hat{R} b_{j} b_{i} \hat{R}^{-1}=\sum_{k, l}^{2 K} b_{l} b_{k}^{2} \mathbf{R}_{k l ; i^{\prime} j^{\prime}}^{*}=\sum_{k, l}^{2 K} b_{l} b_{k}{ }^{2} \mathbf{R}_{i^{\prime} j^{\prime} ; k l}^{\dagger} \quad\left(\forall i^{\prime}, j^{\prime}\right), \\
& \hat{R} b_{j} b_{i} \hat{R}^{-1}=\sum_{k<l}^{2 K} b_{l} b_{k}{ }^{2} \mathcal{R}_{k l ; i^{\prime} j^{\prime}}^{*}=\sum_{k<l}^{2 K} b_{l} b_{k}{ }^{2} \mathcal{R}_{i^{\prime} j^{\prime} ; k l}^{\dagger} \quad\left(i^{\prime}<j^{\prime}\right) .
\end{aligned}
$$

Extension to the general $p$-particle space is straightforward and will not be shown here. Only the symbols ${ }^{p} \mathbf{R}$ and ${ }^{p} \mathcal{R}$ are given for future use.

The operation that transforms the orbital basis induces a transformation in the basis of $p$-electron functions. The chain of $p$ creation operators is in the basis of the $p$ times tensor product of the one-electron creation operator space with itself; the ordered basis is the basis for the antisymmetric part of that tensor product [16]. The matrix transformation is easily constructed as outer direct products from the transformation matrix of the one-electron basis. In what follows, the operators $\hat{R}$, defined through the transformation of the oneelectron basis functions, may appear acting on an $N$-electron function in the same way as the creation and annihilation operators do.

\section{B. The reduced density matrices}

In the second quantization, in the occupation number representation, the $p$-RDM elements may be written as the expectation values of particle-number-conserving operators:

$$
\begin{array}{r}
{ }^{p} D_{i_{1}, i_{2}}, \ldots, i_{p} ; j_{1}, j_{2}, \ldots, j_{p} \\
=\frac{1}{p !}\left\langle\Psi\left|b_{j_{1}}^{\dagger} b_{j_{2}}^{\dagger} \cdots b_{j_{p}}^{\dagger} b_{i_{p}} \cdots b_{i_{2}} b_{i_{1}}\right| \Psi\right\rangle \\
\left(\forall i_{1}, i_{2}, \ldots ; j_{1}, j_{2}, \ldots\right),
\end{array}
$$

where $b_{p}^{\dagger}$ and $b_{i}$ are the creation and annihilation fermion operators, respectively, and the indices represent spin orbitals taken out of a given basis.

As mentioned in the Introduction, when an ordered or antisymmetrized $p$-electron basis is considered, the same information is given in a compressed form:

$$
\begin{aligned}
& { }^{p} \mathcal{D}_{i_{1}, i_{2}}, \ldots, i_{p} ; j_{1}, j_{2}, \ldots, j_{p} \\
& \quad=\left\langle\Psi\left|b_{j_{1}}^{\dagger} b_{j_{2}}^{\dagger} \cdots b_{j_{p}}^{\dagger} b_{i_{p}} \cdots b_{i_{2}} b_{i_{1}}\right| \Psi\right\rangle \\
& \left(\forall i_{1}<i_{2}<\cdots ; j_{1}<j_{2}<\cdots\right) .
\end{aligned}
$$

Both forms of the $p$-RDM have the same trace.

Similar relations hold for the hole RDMs (HRDMs), defined by 


$$
\begin{aligned}
&{ }^{p} \bar{D}_{i_{1}, i_{2}}, \ldots, i_{p} ; j_{1}, j_{2}, \ldots, j_{p} \\
&=\frac{1}{p !}\left\langle\Psi\left|b_{i_{p}} \cdots b_{i_{2}} b_{i_{1}} b_{j_{1}}^{\dagger} b_{j_{2}}^{\dagger} \cdots b_{j_{p}}^{\dagger}\right| \Psi\right\rangle \\
&\left(\forall i_{1}, i_{2}, \ldots ; j_{1}, j_{2}, \ldots\right) .
\end{aligned}
$$

In some cases, an explicit mention of the bra and ket involved in a transition reduced density matrix is required:

$$
{ }^{2} D_{i j ; p q}^{\Phi \Psi}=\frac{1}{2}\left\langle\Psi\left|b_{p}^{\dagger} b_{q}^{\dagger} b_{j} b_{i}\right| \Phi\right\rangle \quad(\forall i, j ; p, q) .
$$

Any of them may also be represented in an ordered basis.

\section{The Hamiltonian}

A Hamiltonian operator, represented in a basis set of $2 \mathrm{~K}$ spin orbitals, that includes, at most, pairwise interactions may be written as

$$
\hat{H}=\frac{1}{2} \sum_{\substack{r, s \\ k, l}}^{2 K}{ }^{0} H_{r s ; k l} b_{r}^{\dagger} b_{s}^{\dagger} b_{l} b_{k},
$$

where ${ }^{0} \mathbf{H}$, of dimensions $(2 K)^{2} \times(2 K)^{2}$, is a self-adjoint numerical matrix, and its element values are given in terms of usual one- and two-electron integrals:

$$
{ }^{0} H_{r s ; k l}=\langle r s \mid k l\rangle+\frac{1}{N-1}\left(h_{r ; k} \delta_{s ; l}+\delta_{r ; k} h_{s ; l}\right) .
$$

The anticommutation relations of the creation and annihilation operators allow us to rearrange the same Hamiltonian in a compressed form [17] in an ordered two-particle basis without loss of information:

$$
\hat{H}=\sum_{\substack{r<s \\ k<l}}^{2 K}{ }^{0} \mathcal{H}_{r s ; k l} b_{r}^{\dagger} b_{s}^{\dagger} b_{l} b_{k}
$$

with

$$
{ }^{0} \mathcal{H}_{r s ; k l}={ }^{0} H_{r s ; k l}-{ }^{0} H_{r s ; l k} .
$$

This Hamiltonian operator depends on the basis set in two ways: through the numerical constants and through the creation-annihilation operators. However, it is as a whole independent of the particular option of the basis set within a given subspace of one-electron functions. So, in the new spin-orbital basis,

$$
\begin{gathered}
\hat{H}=\frac{1}{2} \sum_{\substack{p^{\prime}, q^{\prime} \\
i^{\prime}, j^{\prime}}}^{2 K}{ }^{0} L_{p^{\prime} q^{\prime} ; i^{\prime} j^{\prime}} b_{p^{\prime}}^{\dagger}, b_{q^{\prime}}^{\dagger} b_{j^{\prime}} b_{i^{\prime}} \\
=\sum_{\substack{p^{\prime}<q^{\prime} \\
i^{\prime}<j^{\prime}}}^{2 K}{ }^{0} \mathcal{L}_{p^{\prime} q^{\prime} ; i^{\prime} j^{\prime}} b_{p^{\prime}}^{\dagger} b_{q^{\prime}}^{\dagger}, b_{j^{\prime}} b_{i^{\prime}},
\end{gathered}
$$

where

$$
\begin{aligned}
{ }^{0} L_{p^{\prime} q^{\prime} ; i^{\prime} j^{\prime}}= & \sum_{\substack{r, s \\
k, l}} \sum_{\substack{t, u \\
m, n}}^{2 K}{ }^{2} R_{p^{\prime} q^{\prime} ; t u}^{\dagger}{ }^{0} H_{t u ; m n}{ }^{2} R_{m n ; i^{\prime} j^{\prime}}, \\
{ }^{0} \mathcal{L}_{p^{\prime} q^{\prime} ; i^{\prime} j^{\prime}}= & \sum_{\substack{r<s \\
k<l}} \sum_{\substack{t<u \\
m<n}}^{2 K}{ }^{2} \mathcal{R}_{p^{\prime} q^{\prime} ; t u}^{\dagger}{ }^{0} \mathcal{H}_{t u ; m n}{ }^{2} \mathcal{R}_{m n ; i^{\prime} j^{\prime}}
\end{aligned}
$$

are elements of the matrices ${ }^{0} \mathbf{L}$ and ${ }^{0} \mathcal{L}$, which are respectively the transformed matrices of ${ }^{0} \mathbf{H}$ and ${ }^{0} \mathcal{H}$ to the new basis, and $b_{p^{\prime}}^{\dagger} b_{q^{\prime}}^{\dagger} b_{j^{\prime}} b_{i^{\prime}}$ are creation and annihilation operators referring to the new spin orbitals.

The Hamiltonian is invariant under the transformations of the $\mathrm{U}(2 K)$ group [18]. The change of basis simultaneously transforms the operators and the coefficient matrix ${ }^{0} \mathbf{H}$ into a new matrix ${ }^{0} \mathbf{L}$.

\section{SYMMETRY TRANSFORMATIONS}

Some of the spin-orbital basis transformations just mentioned leave the Hamiltonian matrix and the RDMs invariant, and they will be considered here in some detail.

\section{A. Transformation of the Hamiltonian}

The symmetry properties of the Hamiltonian matrix may be discussed either in the extended form ${ }^{0} \mathbf{H}$ or in the compressed form ${ }^{0} \mathcal{H}$.

Out of the infinite set of transformations of the spinorbital basis, there are some changes of basis $\hat{R}$ for which the associated matrices ${ }^{2} \mathbf{R}$ leave the numerical coefficients, collected in matrix form as ${ }^{0} \mathbf{H}$ or ${ }^{0} \mathbf{L}$, invariant,

$$
{ }^{0} L_{p q ; i j}=\sum_{\substack{m, n \\ t, u}}^{2 K}{ }^{2} R_{p q ; t u}^{\dagger}{ }^{0} H_{t u ; m n}{ }^{2} R_{m n ; i j} \equiv{ }^{0} H_{p q ; i j},
$$

and consequently, the matrix product of ${ }^{2} \mathbf{R}$ and ${ }^{0} \mathbf{H}$ is commutative,

$$
{ }^{2} \mathbf{R}^{0} \mathbf{H}={ }^{0} \mathbf{H}^{2} \mathbf{R}, \quad{ }^{2} \mathcal{R}^{0} \mathcal{H}={ }^{0} \mathcal{H}{ }^{2} \mathcal{R} .
$$

These commutation relations are equivalent to the operator commutation in the first quantization, with a clear distinction: here, the transformation is a change of basis.

They are the only transformations of the one-electron basis, out of the $\mathrm{U}(2 K)$ unitary group, to be considered in this paper. The set of all such transformations $\hat{R}$ forms a group $\mathcal{G}$, a subgroup of $\mathrm{U}(2 K)$ that, for the sake of simplicity, will be taken as finite in what follows. Among these transformations, the point-group symmetries of the molecule may be included. In the first quantization formalism with fixed nuclei, the space symmetry is given by the invariance of the electron-nuclear attraction term under a coordinate system transformation. The symmetry in the occupation number representation may not be the same as in the first quantization, although any judicious choice of a one-electron basis should reflect the space symmetry of the system.

In addition to the spatial symmetry, if no spin interactions are taken into account in the Hamiltonian, any unitary trans- 
formation of the spin parts in the spin orbitals is also a symmetry transformation. In these cases, the symmetry group, considered here, is the direct product of the independent commutative subgroups.

These transformations of the Hamiltonian modify the operators while maintaining the coefficients or vice versa:

$$
\begin{aligned}
\hat{H} & =\sum_{\substack{r, s \\
k, l}}^{2 K} \sum_{\substack{p, q \\
i, j}}^{2 K}{ }^{0} H_{p q ; i j}\left[{ }^{2} R_{i j ; k l}^{\dagger} b_{r}^{\dagger} b_{s}^{\dagger} b_{l} b_{k}{ }^{2} R_{r s ; p q}\right] \\
& =\sum_{\substack{r, s \\
k, l}}^{2 K} \sum_{\substack{p, q \\
i, j}}^{2 K} b_{r}^{\dagger} b_{s}^{\dagger} b_{l} b_{k}\left[{ }^{2} R_{r s ; p q}{ }^{0} H_{p q ; i j} R_{i j ; k l}^{\dagger}\right] .
\end{aligned}
$$

The set of one-particle transformation matrices $\mathbf{R}$ itself constitutes a, most likely reducible, representation $\Gamma$ of the order of $2 K$ of the group; it is generated by the basis of spin orbitals. The set of one-electron creation-annihilation operators generates the same-complex-conjugate representation.

The set of two-particle transformation matrices ${ }^{2} \mathbf{R}$ constitutes another representation: the external product $\Gamma \otimes \Gamma$. The set of matrices ${ }^{2} \mathcal{R}$ is the antisymmetric part of the previous one $\Gamma \otimes \Gamma$.

\section{B. Transformation of the reduced density matrices}

The symmetry properties of the RDMs have been extensively studied [7,19-24] in the coordinate representation as the kernels of integral operators. In what follows, the development will be carried out in the occupation number representation.

As a generalization of what has been said at the end of the preceding section, the set of strings of a larger number of creation-annihilation operators generate powers of the basic $\Gamma$ representation; their ordered subsets generate the corresponding antisymmetric (under permutations of indices) power of $\Gamma$.

Inserting in Eq. (5) the unit operator $\hat{R}^{-1} \hat{R}$ or $\hat{R}^{\dagger} \hat{R}$ at both ends of the chain of creators and annihilators

$$
\begin{aligned}
{ }^{p} D_{i_{1}, i_{2}}, \ldots, i_{p} ; j_{1}, j_{2}, \ldots, j_{p} & \\
= & \frac{1}{p !}\left\langle\Psi\left|\hat{R}^{\dagger} \hat{R} b_{j_{1}}^{\dagger} b_{j_{2}}^{\dagger} \ldots b_{j_{p}}^{\dagger} b_{i_{p}} \ldots b_{i_{2}} b_{i_{1}} \hat{R}^{\dagger} \hat{R}\right| \Psi\right\rangle \\
= & \frac{1}{p !}\left\langle\hat{R} \Psi\left|\hat{R} b_{j_{1}}^{\dagger} b_{j_{2}}^{\dagger} \ldots b_{j_{p}}^{\dagger} b_{i_{p}} \ldots b_{i_{2}} b_{i_{1}} \hat{R}^{\dagger}\right| \hat{R} \Psi\right\rangle,
\end{aligned}
$$

where the operation acting on $N$-electron functions is to be understood as that induced by the transformation of the oneelectron basis. The previous relation shows that the $p$-RDM is invariant under simultaneous transformation of the $\mathrm{N}$-electron state and of the creation-annihilation operators.

\section{Nondegenerate states}

For nondegenerate states $\Psi$ that belong to a onedimensional irreducible representation of the group of trans- formations $\hat{R}$, so that the two-particle associated matrix commutes with ${ }^{0} \mathbf{H}$, the density matrix $|\Psi\rangle\langle\Psi|=| \hat{R} \Psi\rangle\langle\hat{R} \Psi|$ belongs to the trivial, totally symmetric, irreducible representation; and the transformation properties of the RDMs can be analyzed from the transformation of the chains or creation and annihilation operators.

Hence,

$$
{ }^{p} \mathbf{D}={ }^{p} \mathbf{R}^{\dagger p} \mathbf{D}^{p} \mathbf{R}, \quad{ }^{p} \mathcal{D}={ }^{p} \mathcal{R}^{\dagger p} \mathcal{D}^{p} \mathcal{R} \quad(\forall \hat{R} \in \mathcal{G}) .
$$

This is the same type of transformation as that of Eq. (10). For nondegenerate states, the transformations that leave the Hamiltonian matrix invariant, Eq. (12), also leave the $p$-RDM invariant. The $p$-RDM is invariant under its transformation by ${ }^{p} \mathbf{R}$ or, in other words, both matrices commute.

The hole reduced density matrices are also invariant under those symmetry transformations,

$$
{ }^{p} \overline{\mathbf{D}}={ }^{p} \mathbf{R}^{\dagger} p \overline{\mathbf{D}}^{p} \mathbf{R}, \quad{ }^{p} \overline{\mathcal{D}}={ }^{p} \mathcal{R}^{\dagger}{ }^{p} \overline{\mathcal{D}}^{p} \mathcal{R} \quad(\forall R \in \mathcal{G}) .
$$

Powers of $p$-RDMs also transform as the $p$-RDM. For instance,

$$
{ }^{p} \mathbf{D}^{2}={ }^{p} \mathbf{R}^{\dagger p} \mathbf{D}^{p} \mathbf{R}^{p} \mathbf{R}^{\dagger}{ }^{p} \mathbf{D}^{p} \mathbf{R}={ }^{p} \mathbf{R}^{\dagger p} \mathbf{D}^{2}{ }^{p} \mathbf{R}
$$

due to the unitary condition of the ${ }^{p} \mathbf{R}$ matrix.

\section{Degenerate states}

If $\Psi$ forms the basis of the irreducible representation $\mu$ with dimension $l_{\mu}$,

$$
\hat{R} \Psi_{n}^{(\mu)}=\sum_{m=1}^{l_{\mu}} \Psi_{m}^{(\mu)}\left(D^{(\mu)}(R)\right)_{m ; n}, \quad n=1,2, \ldots, l_{\mu} ;
$$

the transformation $\hat{R}$ mixes all the states in a degenerate set under the group $\mathcal{G}$.

The $N$-electron density matrix, associated to one of the states, transforms as

$$
\begin{aligned}
& \left|\hat{R} \Psi_{m}^{(\mu)}\right\rangle\left\langle\hat{R} \Psi_{m}^{(\mu)}\right| \\
& \quad=\sum_{\gamma \eta}^{l_{\mu}}\left(D^{(\mu) \dagger}(R)\right)_{m ; \eta}\left|\Psi_{\gamma}^{(\mu)}\right\rangle\left\langle\Psi_{\eta}^{(\mu)}\right|\left(D^{(\mu)}(R)\right)_{\gamma ; m},
\end{aligned}
$$

but the average density matrix

$$
\frac{1}{l_{\mu}} \sum_{m=1}^{l_{\mu}}\left|\Psi_{m}^{(\mu)}\right\rangle\left\langle\Psi_{m}^{(\mu)}\right|
$$

is totally symmetric under the group $\mathcal{G}$ at the origin of the degeneracy. In other words, if the density matrix transforms as a basis of the totally symmetric representation, its RDMs also transform as a basis of the same totally symmetric representation. This averaged density matrix has a unit trace but it is not idempotent. It corresponds to a state with "less than maximum information" [25]. It has $l_{\mu}$ nonvanishing eigenvalues of value $1 / l_{\mu}$. Its matrix representation in the basis of the eigenstates is invariant under unitary transformations that combine degenerate states among themselves. The reduced 
density matrices that can be obtained from it by contraction are also totally symmetric under the group $\mathcal{G}$.

For instance, if no spin interactions are considered for a set of triplet states, the density matrices of any order, averaged over the states with $M_{S}=1,0,-1$, are invariant under any unitary transformation of the spin parts in the spinorbital basis.

The invariance properties of the $p$-RDMs for nondegenerate states-either particle or hole-given in the preceding subsection, can be extended, in the case of degeneracies, to the average

$$
{ }^{p} \mathbf{D}_{a v}=\frac{1}{l_{\mu}} \sum_{m=1}^{l_{\mu}}{ }^{p} \mathbf{D}^{\Psi_{m} \Psi_{m}},{ }^{p} \overline{\mathbf{D}}_{a v}=\frac{1}{l_{\mu}} \sum_{m=1}^{l_{\mu}}{ }^{p} \overline{\mathbf{D}}^{\Psi_{m} \Psi_{m}} .
$$

In the absence of perturbations, this is the only quantity that can be checked against experimental data. It is an ensemble RDM, with identical statistical weight for every state within the set of degenerate orthonormal states. The average has the same transformation properties as that corresponding to a nondegenerate state.

\section{INVARIANT PARTS OF THE REDUCED DENSITY MATRICES}

As a consequence of relations (13) and (15), i.e., if the system presents symmetries, the information carried by the Hamiltonian matrix ${ }^{0} \mathbf{H}$, as well as that by the RDMs ${ }^{p} \mathbf{D}$ for a nondegenerate state, is multiple times contained and therefore redundant. As a consequence, only a few data, out of a large number of matrix elements, are essential; the rest can be obtained through the symmetry transformations. This number equals the dimension of the subspace spanned by the strings of creation-annihilation operators conserving the number of particles that transform as the totally symmetric irreducible representation. The number of invariants in the Hamiltonian matrix ${ }^{0} \mathbf{H}$ will coincide with that of the 2-RDM for a nondegenerate state.

Let us evaluate now what is the minimum number of data required in order to have all the information contained in the matrices. In order to provide a more transparent description of the procedure, we will consider a specific example: a model molecule made up of six identical atoms, arranged in a plane as a hexagon, and an orbital basis formed by a set of symmetrically orthonormalized real $1 s$ orbitals, one on each atom. For this model system, the spatial symmetry group is finite. The spin part of the basis will be made explicit whenever required to specify different blocks in the RDMs. The RDMs of different orders will be treated independently.

\section{A. The 1-RDM}

The group of spatial transformations and the characters of the representation $\phi$ generated by that basis is presented in the second line of Table I.

The third row shows the characters of the representation $\phi \otimes \phi$ generated by the set of all the products of a creation multiplied by an annihilation operator. It gathers the charac-
TABLE I. Characters of the representation generated by 1-RDM elements

\begin{tabular}{lccccccc}
\hline \hline$C_{6 v}$ & $E$ & $2 C_{6}$ & $2 C_{3}$ & $C_{2}$ & $3 \sigma_{v}$ & $3 \sigma_{d}$ & $g=12$ \\
\hline$\phi$ & 6 & 0 & 0 & 0 & 2 & 0 & \\
$\phi \otimes \phi$ & 36 & 0 & 0 & 0 & 4 & 0 & \\
$\phi \otimes \phi \mid[2]$ & 21 & 0 & 0 & 3 & 5 & 3 & 4 \\
\hline \hline
\end{tabular}

ters of the matrix representation generated by the 36 elements of the 1-RDM.

Due to the Hermiticity of the 1-RDM, only the totally symmetric part under the interchange of rows and columns needs to be taken into account. The characters of the representation generated by those relevant elements are collected in the fourth row. The label [2] appearing in the first column refers to the totally symmetric representation of the permutation group $S_{2}$ [26]. This is clearly a reducible representation, $\Omega$ which may be decomposed into a direct sum of the irreducible representations of the group. Recalling the wellknown formula

$$
a_{A_{1}}=\frac{1}{g} \sum_{\hat{R} \in \mathcal{G}} \chi^{\left(A_{1}\right)}(R)^{*} \chi^{(\Omega)}(R)
$$

where $g$ is the number of operations in the group $\mathcal{G}$, one obtains the number 4 appearing in the last column which denotes the number of times the totally symmetric irreducible representation $A_{1}$, is present in that decomposition. This number indicates the number of invariants; that is, the only nonvanishing data which are able to carry the information of the full matrix.

\section{B. The $p$-RDM}

In the previous example, we have made use of two procedures that are quite general: the construction of the representation generated by the one-body density operator and the calculation of the number of invariants. When considering the $p$-electron space, there is an additional problem: the evaluation of the characters of the representation generated by the strings of $p$ ordered creation operators related to a set of $2 K$ spin orbitals. The representation generated by the strings of annihilators will be its complex conjugate.

In the general case, the $\left(\begin{array}{c}2 K \\ p\end{array}\right)$ different strings of creators constitute a basis for the representation $\Gamma^{\otimes p} \mid\left[1^{p}\right]$, where $\Gamma$ is the basic representation generated by the $2 K$ spin orbitals, and $\left[1^{p}\right]$ indicates that only the totally antisymmetric part of the $p$ th power of the representation is to be considered.

In many problems, it is convenient to specify that the creators and anihilators refer to a spin-adapted basis, i.e., half of the spin orbitals are of the $\alpha$ type and the other half are $\beta$. In this basis, $p$-RDMs for nondegenerate states split into blocks that can be labeled by the quantum numbers $S, M_{S}$ of the $p$-electron basis. For a spin-free Hamiltonian, a further symmetry operation should be considered: the one that interchanges the spin-functions $\alpha$ and $\beta$.

The strings of creators referring to a spin-adapted basis generate the representations indicated in Table II, where $\phi$ is 
TABLE II. Symmetry of the chain of creators.

\begin{tabular}{llll}
\hline \hline Spin function & $S$ & $M_{S}$ & \multicolumn{1}{c}{ Representation } \\
\hline$\alpha^{p}$ & $\frac{p}{2}$ & $\frac{p}{2}$ & $\phi^{\otimes p} \mid\left[1^{p}\right]$ \\
$\alpha^{p-1} \beta$ & & $\frac{p}{2}-1$ & $\phi^{\otimes p-1} \mid\left[1^{p-1}\right] \otimes \phi$ \\
$\alpha^{p-1} \beta$ & $\frac{p}{2}$ & $\frac{p}{2}-1$ & $\phi^{\otimes p} \mid\left[1^{p}\right]$ \\
$\alpha^{p-g 1} \beta$ & $\frac{p}{2}-1$ & $\frac{p}{2}-1$ & $\phi^{\otimes p} \mid\left[2,1^{p-2}\right]$ \\
$\alpha^{p-2} \beta^{2}$ & $\frac{p}{2}-2$ & $\phi^{\otimes p-2}\left|\left[1^{p-2}\right] \otimes \phi^{\otimes 2}\right|\left[1^{2}\right]$ \\
$\alpha^{p-2} \beta^{2}$ & $\frac{p}{2}$ & $\frac{p}{2}-2$ & $\phi^{\otimes p} \mid\left[1^{p}\right]$ \\
$\alpha^{p-2} \beta^{2}$ & $\frac{p}{2}-1$ & $\frac{p}{2}-2$ & $\phi^{\otimes p} \mid\left[2,1^{p-2}\right]$ \\
$\alpha^{p-2} \beta^{2}$ & $\frac{p}{2}-2$ & $\frac{p}{2}-2$ & $\phi^{\otimes p} \mid\left[2^{2}, 1^{p-4}\right]$ \\
$\alpha^{p-3} \beta^{3}$ & $\frac{p}{2}-3$ & $\phi^{\otimes p-3}\left|\left[1^{p-3}\right] \otimes \phi^{\otimes 3}\right|\left[1^{3}\right]$ \\
$\alpha^{p-3} \beta^{3}$ & $\frac{p}{2}$ & $\frac{p}{2}-3$ & $\phi^{\otimes p} \mid\left[1^{p}\right]$ \\
$\alpha^{p-3} \beta^{3}$ & $\frac{p}{2}-1$ & $\frac{p}{2}-3$ & $\phi^{\otimes p} \mid\left[2,1^{p-2}\right]$ \\
$\alpha^{p-3} \beta^{3}$ & $\frac{p}{2}-2$ & $\frac{p}{2}-3$ & $\phi^{\otimes p} \mid\left[2^{2}, 1^{p-4}\right]$ \\
$\alpha^{p-3} \beta^{3}$ & $\frac{p}{2}-3$ & $\frac{p}{2}-3$ & $\phi^{\otimes p} \mid\left[2^{3}, 1^{p-6}\right]$ \\
$\ldots$ & $\cdots$ & $\cdots$ & $\ldots$ \\
\hline \hline
\end{tabular}

the representation of order $K$ generated by the orbital basis. The symmetrized power of a group representation is labeled according to the irreducible representation of the corresponding group of permutations. It expresses that, for a given form of the spin functions, the spatial part should transform as an irreducible representation of the permutation group with no more than two columns in their corresponding Young diagram. For instance, for two electrons $(p=2)$ the $(\alpha \beta)$ twoelectron creators transform as the representation $\phi \otimes \phi$ which splits into triplets $\phi \otimes \phi \mid\left[1^{2}\right]$ and singlets $\phi \otimes \phi \mid[2]$. The same procedure as that described in the 1-RDM case yields the invariants of the various spin blocks of the RDMs. Those of the 2-, 3- and 4-RDMs, for the same model system, are collected in Table III. The first few lines express that the $(\alpha \alpha)$ block $\left(M_{S}=1\right)$ of the 2-RDM for the abovementioned example requires, in a real orbital basis, no more than 16 independent real data as shown in the rightmost column. The third row for every entry in Table III (with label $\mathcal{S}$ $p$-RDM) indicates that, for a symmetric matrix, only the elements in and above the main diagonal are considered. The symmetry due to the interchange of $\alpha$ and $\beta$ parts of the spin orbitals further reduces the number of invariants. The limit is the degrees of freedom of the RDM in any approximation, variational or not.

\section{COMPACT FORMS}

It has previously been shown how the invariances $R$ of the Hamiltonian matrix ${ }^{0} \mathbf{H}$ - and hence of the system-induce invariances in an RDM of any order. The matrix having zero elements everywhere, except for the invariants induced by $R$ in a given ${ }^{p} \mathbf{D}$, is called the compact form of ${ }^{p} \mathbf{D}$ and is denoted by ${ }^{p, c} \mathbf{D}$.

$\mathrm{A}^{p, c} \mathbf{D}$ is a very sparse matrix that nevertheless carries the essential information contained in the corresponding ${ }^{p} \mathbf{D}$.

In this section, we will show how, knowing $R$ and a given ${ }^{p} \mathbf{D}$, one can build a ${ }^{p, c} \mathbf{D}$ and how a ${ }^{p, c} \mathbf{D}$ can be unfolded through the action of $R$ in order to yield back the original ${ }^{p} \mathbf{D}$. It will also be shown here that while a ${ }^{p, c} \mathbf{D}$ yields in a unique way the original ${ }^{p} \mathbf{D}$, several equivalent ${ }^{p, c} \mathbf{D}$ 's may be generated from a given ${ }^{p} \mathbf{D}$.

This folding and unfolding of information can be schematically represented as

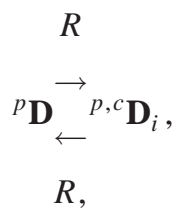

where the " $i$ " index calls our attention to the fact that there is a set of equivalent ${ }^{p, c} \mathbf{D}$.

Note that, in general, one may speak of compact forms of the RDMs, the HRDMs, the Hamiltonian matrix, etc.

The other point of interest, which will be considered here, is that to what extent it is possible to operate directly with these compact forms.

\section{A. Properties of the compact forms}

It has been previously shown that the Hamiltonian matrix (13), the RDMs (15), and the HRDMs (16) must commute with the symmetry transformations. It is possible to obtain from any matrix noncommuting with the symmetry transformations a new matrix that does commute with all of them. The group-theoretical methods show that the operation

$$
\frac{1}{g} \sum_{\hat{R} \in \mathcal{G}}{ }^{p} \mathbf{R}^{p, c} \mathbf{P}^{p} \mathbf{R}^{\dagger}={ }^{p} \mathbf{P}
$$

performed on a matrix ${ }^{p, c} \mathbf{P}$ leads to a new matrix ${ }^{p} \mathbf{P}$ that commutes with every ${ }^{p} \mathbf{S}$ in the group of transformations.

$$
\begin{aligned}
{ }^{p} \mathbf{S}^{p} \mathbf{P}^{p} \mathbf{S}^{\dagger} & ={ }^{p} \mathbf{S}\left[\frac{1}{g} \sum_{\hat{R} \in \mathcal{G}}{ }^{p} \mathbf{R}^{p, c} \mathbf{P}^{p} \mathbf{R}^{\dagger}\right]{ }^{p} \mathbf{S}^{\dagger} \\
& =\frac{1}{g} \sum_{\hat{R} \in \mathcal{G}}{ }^{p}(\mathbf{S R})^{p, c} \mathbf{P}^{p}(\mathbf{S R})^{\dagger}={ }^{p} \mathbf{P} \quad(\forall \hat{S} \in \mathcal{G})
\end{aligned}
$$

and

$$
\begin{aligned}
\frac{1}{g} \sum_{\hat{S} \in \mathcal{G}}{ }^{p} \mathbf{S}\left[\frac{1}{g} \sum_{\hat{R} \in \mathcal{G}}{ }^{p} \mathbf{R}^{p, c} \mathbf{P}^{p} \mathbf{R}^{\dagger}\right]{ }^{p} \mathbf{S}^{\dagger} \\
\quad=\frac{1}{g} \sum_{\hat{S} \hat{R} \in \mathcal{G}}{ }^{p}(\mathbf{S R})^{p, c} \mathbf{P}^{p}(\mathbf{S R})^{\dagger}={ }^{p} \mathbf{P}
\end{aligned}
$$

indicating that it is an idempotent process.

The compact forms ${ }^{p, c} \mathbf{P}$ of the RDMs, HRDMs, or Hamiltonian matrix $(p=2)$ carry the minimum of invariant 
TABLE III. Number of invariants in different spin blocks of 2-, 3-, and 4-RDMs.

\begin{tabular}{|c|c|c|c|c|c|c|c|c|}
\hline Spin blocks of the RDM & Representations & $C_{6 v}: E$ & $2 C_{6}$ & $2 C_{3}$ & $C_{2}$ & $3 \sigma_{v}$ & $3 \sigma_{d}$ & $g=12$ \\
\hline \multirow{3}{*}{$S=1, M_{s}=1$} & $\phi \otimes \phi \mid\left[1^{2}\right]$ & 15 & 0 & 0 & -3 & -1 & -3 & \\
\hline & 2-RDM & 225 & 0 & 0 & 9 & 1 & 9 & \\
\hline & $\mathcal{S}$ 2-RDM & 120 & 0 & 0 & 12 & 8 & 12 & 16 \\
\hline \multirow[t]{3}{*}{$M_{s}=0$} & $\phi \otimes \phi$ & 36 & 0 & 0 & 0 & 4 & 0 & \\
\hline & 2-RDM & 1296 & 0 & 0 & 0 & 16 & 0 & \\
\hline & $\mathcal{S} 2-\mathrm{RDM}$ & 666 & 0 & 0 & 18 & 26 & 18 & 68 \\
\hline \multirow[t]{3}{*}{$S=0, M_{s}=0$} & $\phi \otimes \phi \mid[2]$ & 21 & 0 & 0 & 3 & 5 & 3 & \\
\hline & 2-RDM & 441 & 0 & 0 & 9 & 25 & 9 & \\
\hline & $\mathcal{S}$ 2-RDM & 231 & 0 & 0 & 15 & 23 & 15 & 30 \\
\hline \multirow[t]{3}{*}{$S=3 / 2, M_{s}=3 / 2$} & $\phi^{\otimes 3} \mid\left[1^{3}\right]$ & 20 & 0 & 2 & 0 & -4 & 0 & \\
\hline & 3-RDM & 400 & 0 & 4 & 0 & 16 & 0 & \\
\hline & $\mathcal{S}$ 3-RDM & 210 & 1 & 3 & 10 & 18 & 10 & 26 \\
\hline \multirow[t]{3}{*}{$M_{s}=1 / 2$} & $\phi^{\otimes 2} \mid\left[1^{2}\right] \otimes \phi$ & 90 & 0 & 0 & 0 & -2 & 0 & \\
\hline & 3-RDM & 8100 & 0 & 0 & 0 & 4 & 0 & \\
\hline & $\mathcal{S}$ 3-RDM & 4095 & 0 & 0 & 45 & 47 & 45 & 368 \\
\hline \multirow[t]{3}{*}{$S=1 / 2, M_{s}=1 / 2$} & $\phi^{\otimes 3} \mid[2,1]$ & 70 & 0 & -2 & 0 & 2 & 0 & \\
\hline & 3-RDM & 4900 & 0 & 4 & 0 & 4 & 0 & \\
\hline & $\mathcal{S}$ 3-RDM & 2485 & -1 & 1 & 35 & 37 & 35 & 228 \\
\hline \multirow[t]{3}{*}{$S=2, M_{s}=2$} & $\phi^{\otimes 4} \mid\left[1^{4}\right]$ & 15 & 0 & 0 & 3 & -1 & 3 & \\
\hline & 4-RDM & 225 & 0 & 0 & 9 & 1 & 9 & \\
\hline & $\mathcal{S} 4-\mathrm{RDM}$ & 120 & 0 & 0 & 12 & 8 & 12 & 16 \\
\hline \multirow[t]{3}{*}{$M_{s}=1$} & $\phi^{\otimes 3} \mid\left[1^{3}\right] \otimes \phi$ & 120 & 0 & 0 & 0 & -8 & 0 & \\
\hline & 4-RDM & 14400 & 0 & 0 & 0 & 64 & 0 & \\
\hline & $\mathcal{S} 4$-RDM & 7260 & 0 & 0 & 60 & 92 & 60 & 648 \\
\hline \multirow[t]{3}{*}{$S=1, M_{s}=1$} & $\phi^{\otimes 4} \mid\left[2,1^{2}\right]$ & 105 & 0 & 0 & -3 & -7 & -3 & \\
\hline & 4-RDM & 11025 & 0 & 0 & 9 & 49 & 9 & \\
\hline & $\mathcal{S} 4-\mathrm{RDM}$ & 5565 & 0 & 0 & 57 & 77 & 57 & 502 \\
\hline \multirow[t]{3}{*}{$M_{s}=2$} & $\phi^{\otimes 2}\left|\left[1^{2}\right] \otimes \phi^{\otimes 2}\right|\left[1^{2}\right]$ & 225 & 0 & 0 & 9 & 1 & 9 & \\
\hline & 4-RDM & 50625 & 0 & 0 & 81 & 1 & 81 & \\
\hline & $\mathcal{S} 4-\mathrm{RDM}$ & 25425 & 0 & 0 & 153 & 113 & 153 & 2198 \\
\hline \multirow[t]{3}{*}{$S=0, M_{s}=0$} & $\phi^{\otimes 4} \mid\left[2^{2}\right]$ & 105 & 0 & 0 & 9 & 9 & 9 & \\
\hline & 4-RDM & 11025 & 0 & 0 & 9 & 49 & 9 & \\
\hline & $\mathcal{S} 4-\mathrm{RDM}$ & 5565 & 0 & 0 & 93 & 93 & 93 & 518 \\
\hline
\end{tabular}

information of the respective matrices, but they do not necessarily commute with the transformation matrices ${ }^{p} \mathbf{R}$. However, when a ${ }^{p, c} \mathbf{P}$ is unfolded, the resulting matrix does commute with every ${ }^{p} \mathbf{S}, \hat{S} \in \mathcal{G}$.

The compact form ${ }^{p, c} \mathbf{P}$ may have many elements equal to zero. Their information is accumulated on other elements. The projection brings back every portion of information to its proper place.

The above idea of compactness may be applied to the Hamiltonian matrix

$$
{ }^{0} \mathbf{H}=\frac{1}{g} \sum_{\hat{R} \in \mathcal{G}}{ }^{2} \mathbf{R}^{0, c} \mathbf{H}^{2} \mathbf{R}^{\dagger},
$$

to the RDMs of any order

$$
{ }^{p} \mathbf{D}=\frac{1}{g} \sum_{\hat{R} \in \mathcal{G}}{ }^{p} \mathbf{R}^{p, c} \mathbf{D}^{p} \mathbf{R}^{\dagger},
$$

or to HRDMs

$$
{ }^{p} \overline{\mathbf{D}}=\frac{1}{g} \sum_{\hat{R} \in \mathcal{G}}{ }^{p} \mathbf{R}^{p, c} \overline{\mathbf{D}}^{p} \mathbf{R}^{\dagger}
$$

or to their compressed forms in an ordered $p$-electron basis set. All of them may be expanded from their compact forms.

As an example, consider the particular case of a singlet state studied in the previously mentioned spin-adapted basis. The 1-RDM splits into blocks associated with the $\alpha$ and $\beta$ spins, ${ }^{1} \mathbf{D}^{\alpha}$ and ${ }^{1} \mathbf{D}^{\beta}$, each one of dimensions $K \times K$. In this basis, the mixed blocks vanish. The requirement that the 1-RDM must commute with the transformation that interchanges $\alpha$ and $\beta$ in the spin part of the basis forces the blocks $\alpha$ and $\beta$ to be identical. The group of transformations 
contains only two operations: the identity and the transformation of interchange of $\alpha$ and $\beta,\left(\left\{e, \sigma \leftrightarrow \sigma^{\prime}\right\}\right)$. One of the compact Hermitian forms of the 1-RDM may be

$$
{ }^{1, c} \mathbf{D}^{\sigma}={ }^{1} \mathbf{D}^{\alpha}+{ }^{1} \mathbf{D}^{\beta}, \quad{ }^{1, c} \mathbf{D}^{\sigma^{\prime}}=\mathbf{0},
$$

with all the information compacted in one block. The projec-

$$
\left(\begin{array}{ccc}
1.00000 & 0.66667 & 0 \\
0.66667 & 1.00000 & 0.66667 \\
0 & 0.66667 & 1.00000 \\
-0.33333 & 0 & 0.66667 \\
0 & -0.33333 & 0 \\
0.66667 & 0 & -0.33333
\end{array}\right.
$$

commutes with the transformations contained in the $C_{6 \mathrm{v}}$ group. It may be stored in a compact form as the nonsymmetric matrix

$$
\left(\begin{array}{cccccc}
6 & 0 & 0 & 0 & 0 & 0 \\
8 & 0 & 0 & 0 & 0 & 0 \\
0 & 0 & 0 & 0 & 0 & 0 \\
-2 & 0 & 0 & 0 & 0 & 0 \\
0 & 0 & 0 & 0 & 0 & 0 \\
0 & 0 & 0 & 0 & 0 & 0
\end{array}\right)
$$

From this matrix, the previous one may be recovered by the projection method, Eq. (18), which, in this case, implies the 12 operations of the $C_{6 \mathrm{v}}$ group. Obviously, the higher is the symmetry of a problem, the lesser are the nonzero elements in the compact forms.

Note that, in this example, the $(1,1)$ element of the compact form is the sum of the diagonal elements of the unfolded one because the operations in the group will transfer that value to all places along the diagonal. As an alternative, the same information could have been placed in any diagonal element other than the $(1,1)$. That is, as in many other projection processes, the compact form is not unique. There are many compact forms ${ }^{p, c} \mathbf{P}$ that project to the same complete matrix ${ }^{p} \mathbf{P}$. Indeed, there may be forms more or less compact, with more or less zeros in it.

Since a compact form is not unique, when wishing to construct a compact form one must consider which should be the more convenient distribution of the invariants in the resulting matrix. That is, a preliminary analysis should be carried in which the unfolding process spreads the information contained in one element of the compact form throughout many elements of the expanded form. From this analysis, one can easily deduce where to place the information. tion process will produce the complete 1-RDM. The compact form has many more zeros than the complete matrix. Other spatial symmetries could compact the information even further.

As another simple example, the information contained in the first-order spinless density matrix of a highly symmetric system, such as the benzene molecule, studied under the very simple Hückel method,

$\left.\begin{array}{ccc}-0.33333 & 0 & 0.66667 \\ 0 & -0.33333 & 0 \\ 0.66667 & 0 & -0.33333 \\ 1.00000 & 0.66667 & 0 \\ 0.66667 & 1.00000 & 0.66667 \\ 0 & 0.66667 & 1.00000\end{array}\right)$

The projection procedure outlined above preserves the trace of the matrix,

$$
\operatorname{tr}\left({ }^{p} \mathbf{P}\right)=\operatorname{tr}\left(\frac{1}{g} \sum_{\hat{R} \in \mathcal{G}}{ }^{p} \mathbf{R}^{p, c} \mathbf{P}^{p} \mathbf{R}^{\dagger}\right)=\operatorname{tr}\left({ }^{p, c} \mathbf{P}\right) .
$$

Hence, compact forms have the same trace as the complete matrix.

Compact forms of the Hamiltonian matrix ${ }^{0} \mathbf{H}$, or of the RDMs, can be neither positive semidefinite nor hermitian, although they may be chosen to be Hermitian by the simple method of averaging a matrix and its Hermitian conjugate: $\frac{1}{2}\left({ }^{p, c} \mathbf{P}+{ }^{p, c} \mathbf{P}^{\dagger}\right)$.

The contraction of a compact form of the 2-RDM leads to a compact form of the 1-RDM ${ }^{1, c} \mathbf{D}$ that contains all the information of the 1-RDM:

$$
{ }^{1} \mathbf{D}=\frac{1}{g} \sum_{\hat{R} \in \mathcal{G}} \mathbf{R}^{1, c} \mathbf{D} \mathbf{R}^{\dagger}
$$

Equation (21) is the contraction of Eq. (20) to the oneparticle space. That is, the unfolding of the compact forms is stable under contraction to a subspace with lower number of particles. The compactness is preserved by the contraction process. The same algorithm used to contract any RDM to a lower-order RDM may be used to contract its compact form. Nevertheless, the contraction of a high-order RDM in a compact form does not ensure that what is obtained is the most compact possible form of the lower-order RDM.

The expectation values of operators are calculated as the trace of a product of matrices. The energy is the trace of the product of ${ }^{0} \mathbf{H}$ and ${ }^{2} \mathbf{D}$ matrices: 


$$
\begin{aligned}
E= & \sum_{\substack{k, l \\
r, s}}{ }^{0} H_{r s ; k l}{ }^{2} D_{k l ; r s} \\
= & \sum_{\substack{k, l \\
r, s}}\left[\frac{1}{g} \sum_{\hat{R} \in \mathcal{G}} \sum_{\substack{m, n \\
t, u}}{ }^{2} R_{r s ; m n}{ }^{0, c} H_{m n ; t u}{ }^{2} R_{t u ; k l}^{\dagger}\right] \\
& \times\left[\frac{1}{g} \sum_{\hat{S} \in \mathcal{G}} \sum_{v, w}{ }_{x, y}{ }^{2} S_{k l ; v w}{ }^{2, c} D_{v w ; x y}{ }^{2} S_{x y ; r s}^{\dagger}\right] \\
= & \sum_{\substack{m, n \\
t, u}} 0, c H_{m n ; t u}\left[\frac{1}{g} \sum_{\hat{T} \in \mathcal{G}} \sum_{v, w}{ }^{2}{ }^{2} T_{t u ; y}{ }^{2, c} D_{v w ; x y}{ }^{2} T_{x y ; m n}^{\dagger}\right] .
\end{aligned}
$$

This result can be expressed as the following.

Theorem A. The trace of a product of two matrices is equal to the trace of a product where one of the matrices is in the compact form, while the other matrix is in the unfolded form. The generalization of this theorem is easily proved.

Theorem $B$. The trace of a multiple product of matrices is equal to the trace of a product where all the matrices are in the compact form, except for one of the factors which must be in the unfolded usual form.

These two powerful theorems imply that the use of the compact form matrices greatly enhances the practical possibilities of any methodology when applied to the study of large but highly symmetrical systems.

When approximations or simplifications are required for the study of a large molecular system, it would be simpler to modify_neglecting or approximating, for instance, some elements in ${ }^{0} \mathbf{H}$, while preserving all the symmetries $\hat{R}$ of the original problem-the compact form ${ }^{0, c} \mathbf{H}$ rather than the whole matrix ${ }^{0} \mathbf{H}$. In order to preserve the symmetry of the problem, all the approximations should be consistent with Eq. (13).

Similarly, the RDMs ${ }^{p} \mathbf{D}$ corresponding to the eigenstates are approximated more easily, if in compact form. The number of non-null elements of the most-compact form of an RDM will correspond to the number of degrees of freedom in a variational approximation procedure. This number equals the number of invariants under the transformations $\hat{R}$. Note that here again, in order to preserve the symmetry of the problem, all the approximations should be consistent with Eq. (15).

\section{B. High-order RDMs construction algorithms}

The iterative solution of the CSE requires a reliable calculation of high-order RDMs from RDMs of lower order. Exact algorithms are only known for states described by a single configuration with spin orbitals fully occupied or fully empty. Although many improvements have been achieved in recent times, only approximate algorithms have been proposed for states that take into account the correlation effects.

The basic algorithms proposed include external products of RDMs of low order, antisymmetrized for a fermion problem. These products are Grassman or wedge products [27]. The simplest example is the construction of an approximated
2-RDM from 1-RDM in the form

$$
\begin{array}{r}
{ }^{2} \mathbf{D} \approx{ }^{1} \mathbf{D} \wedge{ }^{1} \mathbf{D},{ }^{2} D_{r s ; k l} \approx \frac{1}{2}\left({ }^{1} D_{r ; k}{ }^{1} D_{s ; l}-{ }^{1} D_{r ; l}{ }^{1} D_{s ; k}\right) \\
(\forall r, s ; k, l) .
\end{array}
$$

The matrices obtained as a result of a Grassman product commute with the matrices ${ }^{p} \mathbf{R}$, associated to the symmetry transformations $\hat{R} \in \mathcal{G}$ if every factor does. That is, the Grassman or the wedge product leads to higher order matrices that have the same symmetry properties as the RDM they try to approximate.

As an extension of the above statement, the cumulants ${ }^{p} \Delta$ that are equated with the difference of an exact (FCI) RDM and an approximation obtained through Grassman products of lower-order RDMs [28-33] are also invariant under transformations that leave the Hamiltonian matrix invariant. That is, the cumulant matrices commute with the symmetry matrix transformations. In the previous example, the second-order cumulant ${ }^{2} \Delta={ }^{2} \mathbf{D}-{ }^{1} \mathbf{D} \wedge{ }^{1} \mathbf{D}$ has the same symmetry properties as the 2-RDM. As in the Hamiltonian matrix or in RDMs, the information contained in cumulants may also be derived from a compact, abbreviated form that reduces or avoids redundancies.

A different problem arises if the information of the loworder matrices is in the compact form mentioned earlier. In general, the Grassman product of low-order compact form RDMs yields a compact-form of the higher-order RDM, which projected on the subspace of matrices that commute with the symmetry transformation matrices, is not coincident with the Grassman product of low-order RDMs in full:

$$
\begin{aligned}
{ }^{2} D_{r s ; k l} \approx & \frac{1}{4} \frac{1}{g^{2}} \sum_{\hat{R}, \hat{S} \in \mathcal{G}}\left[R_{r ; i}^{\dagger} S_{s ; j}^{\dagger}-R_{s ; i}^{\dagger} S_{r ; j}^{\dagger}\right] \\
& \times{ }^{1, c} D_{i ; p}{ }^{1, c} D_{j ; q} \times\left[S_{q ; l} R_{p ; k}-S_{q ; k} R_{p ; l}\right]
\end{aligned}
$$

In other words, the projection of the Grassman products of compact-form RDMs requires an algorithm other than that presented in Eq. (20).

In the case of degeneracies, the use of the average RDM, mentioned in Eq. (17), modifies the equations. The Grassman product of averaged low-order RDMs is not the same as the average of products; there are extra cross terms. This constitutes an alternative approximation of high-order RDMs. But from the point of view of the symmetry, these ensemble RDMs behave as those of a nondegenerate pure state. The Grassman product of averaged RDMs produces a high-order RDM that commutes with the symmetry transformations. On the contrary, the average of wedge products of pure RDMs does not.

\section{Bounds and other corrections}

In addition to the main algorithms for constructing highorder RDMs through Grassman products of lower-order RDMs, a set of corrections have been proposed. All these corrections derive from $N$-representability conditions. Some 
of the conditions affect the entire matrix, but many other are conditions on particular elements of the RDM. The simplest example is that the RDM of any order must be positive semidefinite. Within an orthonormal base, it implies that all the diagonal elements must be non-negative. Hence, zero is the lowest bound for these elements. Other corrections include scaling to adjust the trace, or the partial traces, to known predetermined values. Other bounds are the consequence of the essential non-negativity of the $G$ matrices mentioned by Garrod and Percus [34] and later generalized by Valdemoro et al. [35-37].

These corrections, although rather minor, aim at the precise fulfillment of the known $N$-representability conditions in an attempt to get as close as possible to an exactly $N$-representable RDM.

Rescaling of the entire RDM does not modify its symmetry properties; it commutes with the transformation matrices of the appropriate order. But to rescale only some of the elements, such as the diagonal elements to adjust the trace, may endanger the symmetry properties of the matrix.

All these corrections may be summarized by adding a new matrix and multiplying by a factor, or a combination of both

$$
a\left({ }^{p} \mathbf{D}+\frac{1}{g} \sum_{\hat{R} \in \mathcal{G}}{ }^{p} \mathbf{R}^{p} \mathbf{D}^{\prime}{ }^{p} \mathbf{R}^{\dagger}\right),
$$

where the correction matrix ${ }^{p} \mathbf{D}^{\prime}$ has been projected to ensure that it commutes with every symmetry transformation matrix, and the scaling factor $a$ may be chosen to adjust the trace.

\section{ACKNOWLEDGMENT}

We are greatly indebted to the Spanish Ministerio de Educación, Cultura y Deporte for its support under Project No. BQU2000-1158.
[1] P.O. Löwdin, Phys. Rev. 97, 1474 (1955).

[2] E.R. Davidson, Reduced Density Matrices in Quatum Chemistry (Academic Press, London, 1976).

[3] Reduced Density Matrices with Applications to Physical and Chemical Systems, edited by A.J. Coleman and R.M. Erdahl Queen's Papers on Pure and Applied Mathematics Vol. 11 (Queen's University, Kingston, Ontario, 1968).

[4] Proceedings of the A.J. Coleman Symposium, Kingston, Ontario, 1985, edited by R. Erdahl and V. Smith (Reidel, Dordrecht, 1987).

[5] A.J. Coleman and V.I. Yukalov, Reduced Density Matrices: Coulson's Challenge (Springer, New York, 2000).

[6] C. Garrod, M.V. Mihailovic, and M. Rosina, J. Math. Phys. 16, 868 (1975).

[7] R. McWeeny and Y. Mizuno, Proc. R. Soc. London, Ser. A 259, 554 (1961).

[8] L. Cohen and C. Frishberg, Phys. Rev. A 13, 927 (1976).

[9] H. Nakatsuji, Phys. Rev. A 14, 41 (1976).

[10] F. Colmenero and C. Valdemoro, Int. J. Quantum Chem. 51, 369 (1994).

[11] H. Nakatsuji and K. Yasuda, Phys. Rev. Lett. 76, 1039 (1996).

[12] C. Valdemoro, L.M. Tel, and E. Pérez-Romero, Adv. Quantum Chem. 28, 33 (1997).

[13] D.A. Mazziotti, Int. J. Quantum Chem. 70, 557 (1998).

[14] D.A. Mazziotti, Phys. Rev. A 60, 3618 (1999).

[15] J. Avery, Creation and Annihilation Operators (McGraw-Hill, London, 1976).

[16] Wu-Ki Tung, Group Theory in Physics (World Scientific, Philadelphia, PA, 1985).

[17] J. Applequist, J. Math. Phys. 24, 736 (1983).

[18] P.R. Surján, Second Quantized Approach to Quantum Chemistry. An Elementary Introduction (Springer-Verlag, New York, 1989).
[19] W. Kutzelnigg, Z. Naturforsch. A 18A, 1058 (1963).

[20] R. McWeeny and W. Kutzelnigg, Int. J. Quantum Chem. 2, 187 (1968).

[21] W.A. Bingel and W. Kutzelnigg, Adv. Quantum Chem. 5, 201 (1970).

[22] R.D. Poshusta, J. Math. Phys. 8, 955 (1967).

[23] E.G. Larson, Int. J. Quantum Chem. 13, 121 (1979).

[24] E. Kryachko, Int. J. Quantum Chem. 14, 1 (1981).

[25] U. Fano, Rev. Mod. Phys. 29, 74 (1957).

[26] M.I. Petrashen and E.D. Trifonov, Applications of Group Theory in Quantum Mechanics (MIT Press, Cambridge, MA, 1969).

[27] A.J. Coleman and I. Absar, Int. J. Quantum Chem. 18, 1279 (1980).

[28] W. Kutzelnigg and D. Mukherjee, J. Chem. Phys. 107, 432 (1997).

[29] W. Kutzelnigg and D. Mukherjee, J. Chem. Phys. 110, 2800 (1999).

[30] W. Kutzelnigg and D. Mukherjee, Chem. Phys. Lett. 317, 567 (2000).

[31] D.A. Mazziotti, Phys. Rev. A 57, 4219 (1998).

[32] D.A. Mazziotti, Chem. Phys. Lett. 289, 419 (1998).

[33] P. Ziesche, in Many-Electron Densities and Reduced Density Matrices, edited by J. Cioslowsky (Kluwer Academic, Dodrecht, 2000).

[34] C. Garrod and J.K. Percus, J. Math. Phys. 5, 1756 (1964).

[35] C. Valdemoro, L.M. Tel, and E. Pérez-Romero, Phys. Rev. A 61, 032507 (2000).

[36] C. Valdemoro, L.M. Tel, and E. Pérez-Romero, in ManyElectron Densities and Reduced Density Matrices, edited by J. Cioslowsky (Kluwer Academic, Dordrecht, 2000).

[37] C. Valdemoro, D.R. Alcoba, L.M. Tel, and E. Pérez-Romero, Int. J. Quantum Chem. 85, 214 (2001). 